\title{
On Ideal Convergent Difference Double Sequence Spaces in Intuitionistic Fuzzy Normed Linear Spaces
}

\author{
Esra Kamber ${ }^{1}$ and Selma Altundag $\breve{g}^{1,2 *}$ \\ ${ }^{1}$ Science and Technology Institute, Sakarya University, Sakarya, Turkey \\ ${ }^{2}$ Department of Mathematics, Sakarya University, Sakarya, Turkey \\ ${ }^{*}$ Corresponding author
}

\section{Article Info}

Keywords: Ideal filter, Double Iconvergence, Difference double sequence spaces, Intuitionistic fuzzy normed space

2010 AMS: 40D15, 40G99.

Received: 2 August 2021

Accepted: 1 October 2021

Available online: 1 October 2021

\section{Introduction}

Fuzzy set theory firstly defined by Zadeh [39] has been applied many fields of engineering such as in non-linear dynamic systems [10] , in the population dynamics [5], in the quantum physics [27], but also in various fields of mathematics such as in metric and topological spaces $[7,9,12]$, in the theory of functions [11,38], in the approximation theory [4]. Fuzzy topology plays an essential role in fuzzy theory. It deals with such conditions where the classical theories break down. The intuitionistic fuzzy normed space and intuitionistic fuzzy $n$-normed space which were investigated in [32] and [36] are the most important improvements in fuzzy topology. In the last years, the concepts of intuitionistic fuzzy $I$-convergent difference sequence spaces and intuitionistic fuzzy $I$-convergent difference double sequence spaces have been studied in [21]- [?] and [23]- [24], respectively.

The concept of statistical convergence was given by Steinhaus [34] and Fast [8] using the definition of density of the set of natural numbers. Many years later, statistical convergence was discussed by many researchers in the theory of Fourier analysis, ergodic theory and number theory. Some statistical convergence types were studied in [1]- [3] and [29]. As an extended definition of statistical convergence, definition of $I$-convergence was introduced by Kostyrko, Salat and Wilczynski [26] by using the idea of $I$ of subsets of the set of natural numbers $I$-convergence of double sequences $x=\left(x_{i j}\right)$ has been studied in [30]- [31]. Recently, $I$ - and $I^{*}$ - convergence of double sequences have been studied by Das et. al [6]. Also, related studies can be found in [13]- [17].

Some new sequence spaces were introduced by means of various matrix transformations in [18], [19], [28] and [35]. K1zmaz [25] defined the difference sequence spaces with the difference matrix as follows:

$$
X(\Delta)=\left\{x=\left(x_{k}\right) \in \omega: \Delta x \in X\right\}
$$

for $X=l_{\infty}, c, c_{0}$, where $\Delta x_{k}=x_{k}-x_{k+1}$ and $\Delta$ denotes the difference matrix $\Delta=\left(\Delta_{n k}\right)$ defined by

$$
\Delta_{n k}=\left\{\begin{array}{cl}
(-1)^{n-k}, & \text { if } n \leq k \leq n+1, \\
0, & \text { if } 0 \leq k<n
\end{array}\right.
$$


In this paper, we introduce difference double sequence spaces $I_{2}{ }^{(\mu, v)}(M, \Delta)$ and $I_{2}^{0}(\mu, v)(M, \Delta)$ in the intuitionistic fuzzy normed linear spaces. We also investigate some topological properties of these new spaces.

\section{Basic definitions}

In this section, we give some definitions and notations which will be used for this study.

Definition 2.1. ( [33]) A binary operation $*:[0,1] \times[0,1] \rightarrow[0,1]$ is said to be a continuous $t$-norm if it satisfies the following conditions:

(i) $*$ is associative and commutative,

(ii) $*$ is continuous,

(iii) $a * 1=a$ for all $a \in[0,1]$,

(iv) $a * b \leq c * d$ whenever $a \leq c$ and $b \leq d$ for each $a, b, c, d \in[0,1]$.

Definition 2.2. ( [33]) A binary operation $\circ:[0,1] \times[0,1] \rightarrow[0,1]$ is said to be a continuous $t$-conorm if it satisfies the following conditions:

(i) $\circ$ is associative and commutative,

(ii) $\circ$ is continuous,

(iii) $a \circ 0=a$ for all $a \in[0,1]$,

(iv) $a \circ b \leq c \circ d$ whenever $a \leq c$ and $b \leq d$ for each $a, b, c, d \in[0,1]$.

Definition 2.3. ( [32]) The five-tuple $(X, \mu, v, *, \circ)$ is said to be intuitionistic fuzzy normed linear space (or shortly IFNLS) is where $X$ is a linear space over a field $F, *$ is a continuous $t$-norm, $\circ$ is a continuous $t$-conorm, $\mu, v$ are fuzzy sets on $X \times(0, \infty), \mu$ denotes the degree of membership and $v$ denotes the degree of nonmembership of $(x, t) \in X \times(0, \infty)$ satisfying the following conditions for every $x, y \in X$ and $s, t>0$ :

(i) $\mu(x, t)+v(x, t) \leq 1$,

(ii) $\mu(x, t)>0$,

(iii) $\mu(x, t)=1$ if and only if $x=0$,

(iv) $\mu(\alpha x, t)=\mu\left(x, \frac{t}{|\alpha|}\right)$ if $\alpha \neq 0$,

(v) $\mu(x, t) * \mu(y, s) \leq \mu(x+y, t+s)$,

(vi) $\mu(x,):.(0, \infty) \rightarrow[0,1]$ is continuous,

(vii) $\lim _{t \rightarrow \infty} \mu(x, t)=1$ and $\lim _{t \rightarrow 0} \mu(x, t)=0$,

(viii) $v(x, t)<1$,

(ix) $v(x, t)=0$ if and only if $x=0$,

(x) $v(\alpha x, t)=v\left(x, \frac{t}{|\alpha|}\right)$ if $\alpha \neq 0$,

(xi) $v(x, t) \circ v(y, s) \geq v(x+y, s+t)$,

(xii) $v(x,):.(0, \infty) \rightarrow[0,1]$ is continuous,

(xiii) $\lim _{t \rightarrow \infty} v(x, t)=0$ and $\lim _{t \rightarrow 0} v(x, t)=1$.

In this case $(\mu, v)$ is called intuitionistic fuzzy norm.

Example 2.1. ( [32]) Let $(X,\|\|$.$) be a normed space, and let a * b=a b$ and $a \circ b=\min \{a+b, 1\}$ for all $a, b \in[0,1]$. For all $x \in X$ and every $t>0$, consider

$\mu(x, t):=\frac{t}{t+\|x\|}$ and $v(x, t):=\frac{\|x\|}{t+\|x\|}$

Then $(X, \mu, v, *, \circ)$ is an IFNLS.

Definition 2.4. ( [32]) Let $(X, \mu, v, *, \circ)$ be an IFNLS. For $t>0$, the open ball $B_{x}(r, t)$ with center $x \in X$ and radius $r \in(0,1)$ is defined as

$$
B_{x}(r, t)=\{y \in X: \mu(x-y, t)>1-r \text { and } v(x-y, t)<r\}
$$

Definition 2.5. ( [26]) If $X$ is a non-empty set, then a family of sets $I \subset P(X)$ is called an ideal in $X$ if and only if

(i) $\emptyset \in I$

(ii) $A, B \in I$ implies that $A \cup B \in I$, and

(iii) for each $A \in I$ and $B \subset A$ we have $B \in I$,

where $P(X)$ is the power set of $X$.

Definition 2.6. ( [26]) If $X$ is a non-empty set, then a non-empty family of sets $F \subset P(X)$ is called a filter on $X$ if and only if

(i) $\emptyset \notin F$, 
(ii) $A, B \in F$ implies that $A \cap B \in F$, and

(iii) for each $A \in F$ and $A \subset B$, we have $B \in F$.

An ideal $I$ is called non-trivial if $I \neq \emptyset$ and $X \notin I$. A non-trivial ideal $I \subset P(X)$ is called an admissible ideal in $X$ if and only if it contains all singletons, i.e., if it contains $\{\{x\}: x \in X\}$.

A relation between the concepts of an ideal and a filter is given by the following proposition.

Proposition 2.1. ( [26]) Let $I \subset P(X)$ be a non-trivial ideal. Then the class $F=F(I)=\{M \subset N: M=X-A$, for some $A \in I\}$ is a filter on $X . F=F(I)$ is called the filter associated with the ideal $I$.

Definition 2.7 ( [30]) Let $I_{2}$ be a non-trivial ideal of $N \times N$ and $(X, \mu, v, *, \circ)$ be an IFNLS. A double sequence $x=\left(x_{i j}\right)$ of elements of $X$ is said to be $I_{2}$-convergent to $L \in X$ with respect to the intuitionistic fuzzy linear norm $(\mu, v)$ if, for every $\varepsilon>0$ and $t>0$, the set

$$
\left\{(i, j) \in N \times N: \mu\left(x_{i j}-L, t\right) \leq 1-\varepsilon \text { or } v\left(x_{i j}-L, t\right) \geq \varepsilon\right\} \in I_{2} .
$$

In this case, we write $I_{2}^{(\mu, v)}-\lim x=L$.

Definition 2.9. ( [20]) An Orlicz function is a function $M:[0, \infty) \rightarrow[0, \infty)$ which is continuous, non-decreasing and convex with $M(0)=0$, $M(x)>0$ for $x>0$ and $M(x) \rightarrow \infty$ as $x \rightarrow \infty$. If the convexity of Orlicz function $M$ is replaced by $M(x+y) \leq M(x+y)+M(y)$, then this function is called modulus function.

Remark 2.1. ( [20]) If $M$ is an Orlicz function, then $M(\lambda x) \leq \lambda M(x)$ for all $\lambda$ with $0<\lambda<1$.

\section{Main results}

In this paper, we introduce a variant of ideal convergent difference double sequence spaces in the intuitionistic fuzzy normed linear spaces. We also investigate some topological properties of these new spaces.

Let $w_{2}$ be the space of all double sequences in the intuitionistic fuzzy normed linear spaces. We define the following sequence spaces:

$I_{2}{ }^{(\mu, v)}(M, \Delta)=$

$\left\{\left(x_{i j}\right) \in w_{2}:\left\{(i, j) \in N \times N: M\left(\frac{\mu\left(\Delta x_{i j}-L, t\right)}{\rho}\right) \leq 1-\varepsilon\right.\right.$ or $\left.\left.M\left(\frac{v\left(\Delta x_{i j}-L, t\right)}{\rho}\right) \geq \varepsilon\right\} \in I_{2}\right\}$

and

$I_{2}^{0(\mu, v)}(M, \Delta)=$

$\left\{\left(x_{i j}\right) \in w_{2}:\left\{(i, j) \in N \times N: M\left(\frac{\mu\left(\Delta x_{i j}, t\right)}{\rho}\right) \leq 1-\varepsilon\right.\right.$ or $\left.\left.M\left(\frac{v\left(\Delta x_{i j}, t\right)}{\rho}\right) \geq \varepsilon\right\} \in I_{2}\right\}$.

Theorem 3.1. The spaces $I_{2}{ }^{(\mu, v)}(M, \Delta)$ and $I_{2}^{0^{(\mu, v)}}(M, \Delta)$ are linear spaces.

Proof. We prove the result for $I_{2}{ }^{(\mu, v)}(M, \Delta)$. Similarly, it can be proved for $I_{2}^{0^{(\mu, v)}}(M, \Delta)$. Let $\left(x_{i j}\right),\left(y_{i j}\right) \in I_{2}{ }^{(\mu, v)}(M, \Delta)$ and $\alpha, \beta$ be scalars. The proof is trivial for $\alpha=0$ and $\beta=0$. Let $\alpha \neq 0$ and $\beta \neq 0$. For a given $\varepsilon>0$, choose $s>0$ such that $(1-\varepsilon) *(1-\varepsilon)>1-s$ and $\varepsilon \circ \varepsilon<s$. Hence, we have

$$
\begin{gathered}
A_{1}=\left\{(i, j) \in N \times N: M\left(\frac{\mu\left(\Delta x_{i j}-L_{1}, \frac{t}{2|\alpha|}\right)}{\rho}\right) \leq 1-\varepsilon \text { or } M\left(\frac{v\left(\Delta x_{i j}-L_{1}, \frac{t}{2|\alpha|}\right)}{\rho}\right) \geq \varepsilon\right\} \in I_{2}, \\
A_{2}=\left\{(i, j) \in N \times N: M\left(\frac{\mu\left(\Delta x_{i j}-L_{1}, \frac{t}{2|\beta|}\right)}{\rho}\right) \leq 1-\varepsilon \text { or } M\left(\frac{v\left(\Delta x_{i j}-L_{1}, \frac{t}{2|\beta|}\right)}{\rho}\right) \geq \varepsilon\right\} \in I_{2}, \\
A_{1}^{c}=\left\{(i, j) \in N \times N: M\left(\frac{\mu\left(\Delta x_{i j}-L_{1}, \frac{t}{2|\alpha|}\right)}{\rho}\right)>1-\varepsilon \text { and } M\left(\frac{v\left(\Delta x_{i j}-L_{1}, \frac{t}{2|\alpha|}\right)}{\rho}\right)<\varepsilon\right\} \in F\left(I_{2}\right),
\end{gathered}
$$

and

$$
A_{2}^{c}=\left\{(i, j) \in N \times N: M\left(\frac{\mu\left(\Delta x_{i j}-L_{1}, \frac{t}{2|\beta|}\right)}{\rho}\right)>1-\varepsilon \text { and } M\left(\frac{v\left(\Delta x_{i j}-L_{1}, \frac{t}{2|\beta|}\right)}{\rho}\right)<\varepsilon\right\} \in F\left(I_{2}\right) .
$$

Let define the set $A_{3}=A_{1} \cup A_{2}$. Hence $A_{3} \in I_{2}$. It follows that $A_{3}^{c}$ is a non-empty set in $F\left(I_{2}\right)$. We will prove that for every $\left(x_{i j}\right),\left(y_{i j}\right) \in$ $I_{2}{ }^{(\mu, v)}(M, \Delta)$

$A_{3}^{c} \subset\left\{(i, j) \in N \times N: M\left(\frac{\mu\left(\left(\alpha . \Delta x_{i j}+\beta . \Delta y_{i j}\right)-\left(\alpha . L_{1}+\beta . L_{2}\right), t\right)}{\rho}\right)>1-s\right.$

and $\left.M\left(\frac{v\left(\left(\alpha . \Delta x_{i j}+\beta . \Delta y_{i j}\right)-\left(\alpha \cdot L_{1}+\beta . L_{2}\right), t\right)}{\rho}\right)<s\right\}$. 
Let $(m, n) \in A_{3}^{c}$. In this case,

$M\left(\frac{\mu\left(\Delta x_{m n}-L_{1}, \frac{t}{2|\alpha|}\right)}{\rho}\right)>1-\varepsilon$ and $M\left(\frac{v\left(\Delta x_{m n}-L_{1}, \frac{t}{2|\alpha|}\right)}{\rho}\right)<\varepsilon$,

and

$M\left(\frac{\mu\left(\Delta y_{m n}-L_{2}, \frac{t}{2|\beta|}\right)}{\rho}\right)>1-\varepsilon$ and $M\left(\frac{v\left(\Delta y_{m n}-L_{2}, \frac{t}{2|\beta|}\right)}{\rho}\right)<\varepsilon$.

Then

$$
\begin{gathered}
M\left(\frac{\mu\left(\left(\alpha . \Delta x_{m n}+\beta . \Delta y_{m n}\right)-\left(\alpha . L_{1}+\beta . L_{2}\right), t\right)}{\rho}\right) \\
\geq M\left(\frac{\mu\left(\alpha . \Delta x_{m n}-\alpha . L_{1}, t / 2\right)}{\rho}\right) * M\left(\frac{\mu\left(\beta . \Delta y_{m n}-\beta . L_{2}, t / 2\right)}{\rho}\right)
\end{gathered}
$$

$=M\left(\frac{\mu\left(\Delta x_{m n}-L_{1}, \frac{t}{2|\alpha|}\right)}{\rho}\right) * M\left(\frac{\mu\left(\Delta y_{m n}-L_{2}, \frac{t}{2|\beta|}\right)}{\rho}\right)>(1-\varepsilon) *(1-\varepsilon)>1-s$

and

$$
\begin{gathered}
M\left(\frac{v\left(\left(\alpha . \Delta x_{m n}+\beta . \Delta y_{m n}\right)-\left(\alpha . L_{1}+\beta . L_{2}\right), t\right)}{\rho}\right) \\
\leq M\left(\frac{v\left(\alpha . \Delta x_{m n}-\alpha \cdot L_{1}, t / 2\right)}{\rho}\right) \circ M\left(\frac{v\left(\beta . \Delta y_{m n}-\beta . L_{2}, t / 2\right)}{\rho}\right)
\end{gathered}
$$

$=M\left(\frac{v\left(\Delta x_{m n}-L_{1}, \frac{t}{2|\alpha|}\right)}{\rho}\right) \circ M\left(\frac{v\left(\Delta y_{m n}-L_{2}, \frac{t}{2|\beta|}\right)}{\rho}\right)<\varepsilon \circ \varepsilon<s$.

This proves that

$A_{3}^{c} \subset\left\{(i, j) \in N \times N: M\left(\frac{\mu\left(\left(\alpha . \Delta x_{i j}+\beta . \Delta y_{i j}\right)-\left(\alpha . L_{1}+\beta . L_{2}\right), t\right)}{\rho}\right)>1-s\right.$

and $\left.M\left(\frac{v\left(\left(\alpha . \Delta x_{i j}+\beta . \Delta y_{i j}\right)-\left(\alpha \cdot L_{1}+\beta . L_{2}\right), t\right)}{\rho}\right)<s\right\}$.

Hence $I_{2}{ }^{(\mu, v)}(M, \Delta)$ is a linear space.

Theorem 3.2. Every closed ball $B_{x}^{c}(r, t)(M)$ is an open set in $I_{2}{ }^{(\mu, v)}(M, \Delta)$.

Proof. Let $B_{x}(r, t)(M)$ be an open ball with centre $x \in I_{2}{ }^{(\mu, v)}(M, \Delta)$ and radius $r \in(0,1)$ with respect to $t$, i.e.

$B_{x}(r, t)(M)=\left\{y \in I_{2}{ }^{(\mu, v)}(M, \Delta):\right.$

$\left\{(i, j) \in N \times N: M\left(\frac{\mu\left(\Delta x_{i j}-\Delta y_{i j}, t\right)}{\rho}\right) \leq 1-r\right.$ or $\left.\left.M\left(\frac{\mu\left(\Delta x_{i j}-\Delta y_{i j}, t\right)}{\rho}\right) \geq r\right\} \in I_{2}\right\}$.

Let $y \in B_{x}^{c}(r, t)(M)$. So $M\left(\frac{\mu(\Delta x-\Delta y, t)}{\rho}\right)>1-r$ and $M\left(\frac{v(\Delta x-\Delta y, t)}{\rho}\right)<r$.

Since $M\left(\frac{\mu(\Delta x-\Delta y, t)}{\rho}\right)>1-r$, there exists $t_{0} \in(0, t)$ such that $M\left(\frac{\mu\left(\Delta x-\Delta y, t_{0}\right)}{\rho}\right)>1-r$ and $M\left(\frac{v\left(\Delta x-\Delta y, t_{0}\right)}{\rho}\right)<r$.

Let $r_{0}=M\left(\frac{\mu\left(\Delta x-\Delta y, t_{0}\right)}{\rho}\right)$. Since $r_{0}>1-r$, there exists $s \in(0,1)$ such that $r_{0}>1-s>1-r$ and so there exists $r_{1}, r_{2} \in(0,1)$ such that $r_{0} * r_{1}>1-s$ and $\left(1-r_{0}\right) \circ\left(1-r_{2}\right)<s$.

Let $r_{3}=\max \left\{r_{1}, r_{2}\right\}$. Then $1-s<r_{0} * r_{1} \leq r_{0} * r_{3}$ and $\left(1-r_{0}\right) \circ\left(1-r_{3}\right) \leq\left(1-r_{0}\right) \circ\left(1-r_{2}\right)<s$.

Consider the closed balls $B_{y}^{c}\left(1-r_{3}, t-t_{0}\right)(M)$ and $B_{x}^{c}(r, t)(M)$. We prove that $B_{y}^{c}\left(1-r_{3}, t-t_{0}\right)(M) \subset B_{x}^{c}(r, t)(M)$. Let $z \in B_{y}^{c}\left(1-r_{3}, t-\right.$ 
$\left.t_{0}\right)(M)$. Then $M\left(\frac{\mu\left(\Delta y-\Delta z, t-t_{0}\right)}{\rho}\right)>r_{3}$ and $M\left(\frac{v\left(\Delta y-\Delta z, t-t_{0}\right)}{\rho}\right)<1-r_{3}$. Hence

$M\left(\frac{\mu(\Delta x-\Delta z, t)}{\rho}\right) \geq M\left(\frac{\mu\left(\Delta x-\Delta y, t_{0}\right)}{\rho}\right) * M\left(\frac{\mu\left(\Delta y-\Delta z, t-t_{0}\right)}{\rho}\right)>r_{0} * r_{3} \geq r_{0} * r_{1}>1-s>1-r$,

and

$M\left(\frac{v(\Delta x-\Delta z, t)}{\rho}\right) \leq M\left(\frac{v\left(\Delta x-\Delta y, t_{0}\right)}{\rho}\right) \circ M\left(\frac{v\left(\Delta y-\Delta z, t-t_{0}\right)}{\rho}\right)$

$<\left(1-r_{0}\right) \circ\left(1-r_{3}\right)<s<r$.

Thus $z \in B_{x}^{c}(r, t)(M)$ and it proves that $B_{y}^{c}\left(1-r_{3}, t-t_{0}\right)(M) \subset B_{x}^{c}(r, t)(M)$.

Remark 3.1. It is clear that $I_{2}{ }^{(\mu, v)}(M, \Delta)$ is an IFNLS. Define

$\tau_{2}{ }^{(\mu, v)}(M, \Delta)=\left\{A \subset I_{2}{ }^{(\mu, v)}(M, \Delta):\right.$

for each $x \in A$, there exist $t>0$ and $r \in(0,1)$ such that $\left.B_{x}^{c}(r, t)(M) \subset A\right\}$.

Then $\tau_{2}{ }^{(\mu, v)}(M, \Delta)$ is a topology on $I_{2}{ }^{(\mu, v)}(M, \Delta)$.

Theorem 3.3. The topology $\tau_{2}^{(\mu, v)}(M, \Delta)$ on $I_{2}^{0(\mu, v)}(M, \Delta)$ is first countable.

Proof. It is clear that $\left\{B_{x}^{c}\left(\frac{1}{n}, \frac{1}{n}\right)(M): n \in N\right\}$ is a local base at $x \in I_{2}{ }^{(\mu, v)}(M, \Delta)$. Then, the topology $\tau_{2}{ }^{(\mu, v)}(M, \Delta)$ on $I_{2}^{0}(\mu, v)(M, \Delta)$ is first countable.

Theorem 3.4. $I_{2}{ }^{(\mu, v)}(M, \Delta)$ and $I_{2}^{0}{ }^{(\mu, v)}(M, \Delta)$ are Hausdorff spaces.

Proof. Let $x, y \in I_{2}{ }^{(\mu, v)}(M, \Delta)$ such that $x \neq y$. Then $0<M\left(\frac{\mu(\Delta x-\Delta y, t)}{\rho}\right)<1$ and $0<M\left(\frac{v(\Delta x-\Delta z, t)}{\rho}\right)<1$.

Define $r_{1}, r_{2}$ and $r$ such that $r_{1}=M\left(\frac{\mu(\Delta x-\Delta y, t)}{\rho}\right), r_{2}=M\left(\frac{v(\Delta x-\Delta y, t)}{\rho}\right)$ and $r=\max \left\{r_{1}, 1-r_{2}\right\}$. Then for each $r_{0} \in(r, 1)$ there exist $r_{3}$ and $r_{4}$ such that $r_{3} * r_{4} \geq r_{0}$ and $\left(1-r_{3}\right) \circ\left(1-r_{4}\right) \leq\left(1-r_{0}\right)$.

Let $r_{5}=\max \left\{r_{3},\left(1-r_{4}\right)\right\}$ and consider the closed balls $B_{x}^{c}\left(1-r_{5}, \frac{t}{2}\right)(M)$ and $B_{y}^{c}\left(1-r_{5}, \frac{t}{2}\right)(M)$. Then, clearly $B_{x}^{c}\left(1-r_{5}, \frac{t}{2}\right)(M) \cap B_{y}^{c}(1-$ $\left.r_{5}, \frac{t}{2}\right)(M)=\emptyset$.

Suppose that $z \in B_{x}^{c}\left(1-r_{5}, \frac{t}{2}\right)(M) \cap B_{y}^{c}\left(1-r_{5}, \frac{t}{2}\right)(M)$. So,

$r_{1}=M\left(\frac{\mu(\Delta x-\Delta y, t)}{\rho}\right) \geq M\left(\frac{\mu(\Delta x-\Delta z, t / 2)}{\rho}\right) * M\left(\frac{\mu(\Delta y-\Delta z, t / 2)}{\rho}\right)$

$\geq r_{5} * r_{5} \geq r_{3} * r_{4} \geq r_{0}>r$ and

$r_{2}=M\left(\frac{v(\Delta x-\Delta y, t)}{\rho}\right) \leq M\left(\frac{v(\Delta x-\Delta z, t / 2)}{\rho}\right) \circ M\left(\frac{v(\Delta y-\Delta z, t / 2)}{\rho}\right)$

$\leq\left(1-r_{5}\right) \circ\left(1-r_{5}\right) \leq\left(1-r_{3}\right) \circ\left(1-r_{4}\right) \leq\left(1-r_{0}\right)<1-r$,

which is a contradiction. Hence $I_{2}{ }^{(\mu, v)}(M, \Delta)$ is a Hausdorff space.

Theorem 3.5. Let $I_{2}{ }^{(\mu, v)}(M, \Delta)$ be an IFNLS, $\tau_{2}{ }^{(\mu, v)}(M, \Delta)$ be a topology on $I_{2}{ }^{(\mu, v)}(M, \Delta)$ and $\left(x_{i j}\right)$ be a sequence in $I_{2}{ }^{(\mu, v)}(M, \Delta)$. Then a sequence $\left(x_{i j}\right)$ is $\Delta$-convergent to $\Delta x_{0}$ with respect to the intuitionistic fuzzy linear norm $(\mu, v)$ if and only if $M\left(\frac{\mu\left(\Delta x_{i j}-\Delta x_{0}, t\right)}{\rho}\right) \longrightarrow 1$ and $M\left(\frac{v\left(\Delta x_{i j}-\Delta x_{0}, t\right)}{\rho}\right) \longrightarrow 0$ as $i, j \longrightarrow \infty$.

Proof. Let $B_{x_{0}}(r, t)(M)$ be an open ball with centre $x_{0} \in I_{2}{ }^{(\mu, v)}(M, \Delta)$ and radius $r \in(0,1)$ with respect to $t$, i.e.

$B_{x_{0}}(r, t)(M)=\left\{\left(x_{i j}\right) \in I_{2}{ }^{(\mu, v)}(M, \Delta):\right.$

$\left\{(i, j) \in N \times N: M\left(\frac{\mu\left(\Delta x_{i j}-\Delta x_{0}, t\right)}{\rho}\right) \leq 1-r\right.$ or $\left.\left.M\left(\frac{\mu\left(\Delta x_{i j}-\Delta x_{0}, t\right)}{\rho}\right) \geq r\right\} \in I_{2}\right\}$.

Suppose $\left(x_{i j}\right)$ is $\Delta$-convergent to $\Delta x_{0}$ with respect to the intuitionistic fuzzy linear norm $(\mu, v)$. Then for $r \in(0,1)$ and $t>0$, there exists $k_{0} \in N$ such that $\left(x_{i j}\right) \in B_{x_{0}}^{c}(r, t)(M)$ for all $i, j \geq k_{0}$. Thus,

$\left\{(i, j) \in N \times N: M\left(\frac{\mu\left(\Delta x_{i j}-\Delta x_{0}, t\right)}{\rho}\right)>1-r\right.$ and $\left.M\left(\frac{v\left(\Delta x_{i j}-\Delta x_{0}, t\right)}{\rho}\right)<r\right\} \in F\left(I_{2}\right)$. 

So $1-M\left(\frac{\mu\left(\Delta x_{i j}-\Delta x_{0}, t\right)}{\rho}\right)<r$ and $M\left(\frac{v\left(\Delta x_{i j}-\Delta x_{0}, t\right)}{\rho}\right)<r$ for all $i, j \geq k_{0}$. Then $M\left(\frac{\mu\left(\Delta x_{i j}-\Delta x_{0}, t\right)}{\rho}\right) \longrightarrow 1$ and $M\left(\frac{v\left(\Delta x_{i j}-\Delta x_{0}, t\right)}{\rho}\right) \longrightarrow$
0 as $i, j \longrightarrow \infty$.

Conversely, if for each $t>0$,

$M\left(\frac{\mu\left(\Delta x_{i j}-\Delta x_{0}, t\right)}{\rho}\right) \longrightarrow 1$ and $M\left(\frac{v\left(\Delta x_{i j}-\Delta x_{0}, t\right)}{\rho}\right) \longrightarrow 0$ as $i, j \longrightarrow \infty$. Then for $r \in(0,1)$, there exists $k_{0} \in N$ such that $1-M\left(\frac{\mu\left(\Delta x_{i j}-\Delta x_{0}, t\right)}{\rho}\right)<$ $r$ and $M\left(\frac{v\left(\Delta x_{i j}-\Delta x_{0}, t\right)}{\rho}\right)<r$ for all $i, j \geq k_{0}$. So, $M\left(\frac{\mu\left(\Delta x_{i j}-\Delta x_{0}, t\right)}{\rho}\right)>1-r$ and $M\left(\frac{v\left(\Delta x_{i j}-\Delta x_{0}, t\right)}{\rho}\right)<r$ for all $i, j \geq k_{0}$. Hence $\left(x_{i j}\right) \in B_{x_{0}}^{c}(r, t)(M)$ for all $i, j \geq k_{0}$. This proves that a sequence $\left(x_{i j}\right)$ is $\Delta$-convergent to $\Delta x_{0}$ with respect to the intuitionistic fuzzy linear $\operatorname{norm}(\mu, v)$.

\section{Acknowledgements}

The authors would like to express their sincere thanks to the editor and the anonymous reviewers for their helpful comments and suggestions.

\section{Funding}

There is no funding for this work.

\section{Availability of data and materials}

Not applicable.

\section{Competing interests}

The authors declare that they have no competing interests.

\section{Author's contributions}

All authors contributed equally to the writing of this paper. All authors read and approved the final manuscript.

\section{References}

[1] S. Altundağ, E. Kamber, Weighted statistical convergence in intuitionistic fuzzy normed linear spaces, J. Inequal. Spec. Funct., 8(2017), 113-124.

[2] S. Altundağ, E. Kamber, Weighted lacunary statistical convergence in intuitionistic fuzzy normed linear spaces, Gen. Math. Notes, 37(2016), 1-19.

[3] S. Altundağ, E. Kamber, Lacunary $\Delta$-statistical convergence in intuitionistic fuzzy n-normed linear spaces, Journal of inequalities and applications, 40(2014), 1-12.

[4] G.A. Anastassiou, Fuzzy approximation by fuzzy convolution type operators, Comput. Math. Appl., 48(2004), $1369-1386$.

[5] L.C. Barros, R.C. Bassanezi, P.A. Tonelli, Fuzzy modelling in population dynamics, Ecol. Model., 128(2000), $27-33$.

[6] P. Das, P. Kostyrko, W. Wilczynski, P. Malik, I- and I*- convergence of double sequences, Math. Slovaca, 58(2008), 605-620.

[7] M.A. Erceg, Metric spaces in fuzzy set theory, J. Math. Anal. Appl., 69(1979), 205-230.

[8] H. Fast, Sur la convergence statistique, Colloq. Math., 2(1951), 241-244.

[9] A. George, P. Veeramani, On some result in fuzzy metric space, Fuzzy Sets Syst., 64(1994), 395-399.

[10] L. Hong, J.Q. Sun, Bifurcations of fuzzy nonlinear dynamical systems, Commun. Nonlinear Sci. Numer. Simul., 1(2006), 1-12.

[11] G. Jäger, Fuzzy uniform convergence and equicontinuity, Fuzzy Sets Syst., 109(2000), 187-198.

[12] O. Kaleva, S. Seikkala, On fuzzy metric spaces, Fuzzy Sets Syst., 12(1984), 215-229.

[13] V.A. Khan, R.K.A. Rababah, K.M.A.S. Alshlool, S.A.A. Abdullah, A. Ahmad, On ideal convergence Fibonacci difference sequence spaces, Adv. Differ. Equ.,2018(2018), 1-14.

[14] V.A. Khan, M. Ahmad, H. Fatima, M.F. Khan, On some results in intuitionistic fuzzy ideal convergence double sequence spaces, Adv. Differ. Equ., $\mathbf{2 0 1 9}(2019), 1-10$.

[15] V. Kumar, K. Kumar, On ideal convergence of double sequences in intuitionistic fuzzy normed spaces, Selçuk J. Appl. Math. 24(2009), 27-41.

[16] A. K $\ddot{A} \pm 1 \ddot{A} \pm \tilde{A} \S m a n, S$. Borgohain, Generalized difference strongly summable sequence spaces of fuzzy real numbers defined by ideal convergence and Orlicz function, Adv. Differ. Equ., 2013(2013), 1-10.

[17] S.A. Mohiuddine, A. Alotaibi, S.M. Alsulami,Ideal convergence of double sequences in random 2-normed spaces, Adv. Differ. Equ.,2012(2012), 1-8.

[18] E.E. Kara,Some topological and geometrical properties of new Banach sequence spaces, J. Inequal. Appl.,2013(2013), 1-15.

[19] E.E. Kara, M. Ilkhan, On some Banach sequence spaces derived by a new band matrix, Br. J. Math. Comput. Sci., 9(2015), 141 â€“159.

[20] E.E. Kara, M. Ilkhan, Lacunary I-convergent and lacunary I-bounded sequence spaces defined by an Orlicz function, Electronic Journal of Mathematical Analysis and Applications, 4(2016), 150-159.

[21] E. Kamber, Intuitionisic fuzzy I-convergent difference sequence spaces defined by modulus function, J. Inequal. Spec. Funct., 10(2019), 93-100.

[22] E. Kamber, Intuitionisic fuzzy I-convergent difference sequence spaces defined by compact operator, submitted.

[23] E. Kamber, Intuitionisic fuzzy I-convergent difference double sequence spaces, Research and Communications in Mathematics and Mathematical Sciences, 10(2018), 141-153.

[24] E. Kamber, S. Altundağ, Intuitionisic fuzzy I-convergent difference double sequence spaces defined by modulus function, submitted

[25] H. Kizmaz, On certain sequence spaces, Canad. Math. Bull., 24(1981), 169-176.

[26] P. Kostyrko, T. Salat, W. Wilczynski, I-convergence, Real Analysis Exchange, 26(2000), 669-686.

[27] J. Madore, Fuzzy physics, Ann. Phys., 219(1992), 187-198.

[28] E. Malkowsky, Recent results in the theory of matrix transformation in sequence spaces, Math. Vesnik, 49(1997), 187-196.

[29] M.A. Tok, E.E. Kara, S. Altundağ, On the $\alpha \beta$-statistical convergence of the modified discrete operators, Adv. Diff.Equ.,2018(2018), 1-6.

[30] M. Mursaleen, S.A. Mohiuddine, H. Osama, H. Edely, On the ideal convergence of double sequences in intuitionistic fuzzy normed spaces, Computers and Mathematics with Applications, 59(2010), 603-611. 
[31] E. Dündar, U. Ulusu, N. Pancaroğlu, Strongly I2-lacunary convergence and $I_{2}$-lacunary Cauchy double sequences of sets, Aligarh Bull. Math., 35(2016),

[32] R. Saadati, J.H. Park, On the intuitionistic fuzzy topological spaces, Chaos Soliton. Fractal., 27(2006), $331-344$.

33] B. Schweizer, A. Sklar, Statistical metric spaces, Pac. J. Math., 10(1960), 313-334.

[34] H. Steinhaus, Sur la convergence ordinaire et la convergence asymptotique, Colloq. Math., 2(1951), 73-74.

[35] Şengönül, M., On the Zweier sequence space, Demonstratio Mathematica, XL(2007), 181-196.

[36] S. Vijayabalaji, , N. Thillaigovindan, Y.B. Jun, Intuitionistic fuzzy n- normed linear space, Bull. Korean. Math. Soc., 44(2007), 291-308.

[37] C. S. Wang, On Nörlund sequence spaces, Tamkang J. Math., 9(1978), 269-274.

[38] K. Wu, Convergences of fuzzy sets based on decomposition theory and fuzzy polynomial function, Fuzzy Sets Syst., 109(2000), 173-185.

[39] Zadeh, L.A., Fuzzy Sets, Inform. Cont., 8(1965). 